\title{
Calibration and monitoring units of the Baikal-GVD neutrino telescope
}

A.D. Avrorin ${ }^{a}$, A.V. Avrorin ${ }^{a}$, V.M. Aynutdinov ${ }^{a}$, R. Bannash ${ }^{g}$, I.A. Belolaptikov ${ }^{b}$, V.B. Brudanin ${ }^{b}$, N.M. Budnev ${ }^{c}$, I.A. Danilchenko ${ }^{a}$, G.V. Domogatsky, A.A.Doroshenko ${ }^{a}$, R. Dvornickýb,h A.N. Dyachok ${ }^{c}$, Zh.-A.M. Dzhilkibaev ${ }^{a}$, L. Fajt ${ }^{b, h, i}$, S.V. Fialkovsky ${ }^{e}$, A.R. Gafarov ${ }^{c}$, K.V. Golubkov ${ }^{a}$, T.I. Gress ${ }^{c}$, Z. Honz ${ }^{b}$, K.G.Kebkal ${ }^{g}$, O.G. Kebkal ${ }^{g}$, M.M. Kolbin ${ }^{b}$, K.V. Konischev ${ }^{b}$, A.V. Korobchenko ${ }^{b}$, A.P.Koshechkin ${ }^{a}$, F.K. Koshel ${ }^{a}$, A.V. Kozhin ${ }^{d}$, V.F. Kulepov ${ }^{e}$, D.A. Kuleshov ${ }^{a}$, M.B.Milenin ${ }^{e}$, R.A.Mirgazov ${ }^{c}$, E.R. Osipova ${ }^{d}$, A.I. Panfilov', L.V. Pan'kov ${ }^{c}$, D.P.Petukhov ${ }^{a}$, E.N.Pliskovsky ${ }^{b}$, M.I., Rozanov ${ }^{f}$, E.V. Rjabov ${ }^{c}$, G.B.Safronov ${ }^{b}$, B.A.Shaybonov ${ }^{b}$, M.D.Shelepov ${ }^{a}$, F. Simkovic ${ }^{b, h, i}$, A.V. Skurikhin ${ }^{d}$, I.StekI ${ }^{i}$, O.V. Suvorova ${ }^{a}$, V.A., Tabolenko ${ }^{c}$, B.A.Tarashansky ${ }^{c}$, S.A.Yakovlev ${ }^{g}$, A.V.Zagorodnikov $^{c}$ and V.L. Zurbanov ${ }^{c}$

${ }^{a}$ Institute for Nuclear Research, Moscow, 117312 Russia

${ }^{b}$ Joint Institute for Nuclear Research, Dubna, 141980 Russia

'Irkutsk State University, Irkutsk, 664003 Russia

${ }^{d}$ Institute of Nuclear Physics, Moscow State University, Moscow, 119991 Russia

${ }^{e}$ Nizhni Novgorod State Technical University, Nizhni Novgorod, 603950 Russia

${ }^{f}$ St. Petersburg State Marine Technical University, St. Petersburg, 190008 Russia

${ }^{g}$ EvoLogics, Germany

${ }^{h}$ Comenius University, Bratislava, Slovakia

${ }^{i}$ Czech Technical University in Prague, Prague, Czech Republic

\begin{abstract}
The Baikal-GVD is a cubic kilometer scale neutrino telescope which is deployed in Lake Baikal, that will perform neutrino astronomy studies. It consists of sub-arrays - clusters of about 300 optical modules each, that detect the Cherenkov light radiated by charged particles induced by neutrino interactions with the surrounding medium. The performance of the neutrino telescope relies on the precise timing and positioning calibration of the detector elements, continuous control and monitoring of a behavior of measuring systems, as well as environmental conditions which may affect light detection and event selection. This contribution describes the units which are used in GVD for calibration and monitoring purposes.
\end{abstract}

35th International Cosmic Ray Conference - ICRC2017

10-20 July, 2017

Bexco, Busan, Korea

${ }^{1}$ Speaker, e-mail: k.golubkov@gmail.com 


\section{Introduction}

The current design of Giga tons volume detector the Baikal-GVD (NT-1000) [1],[2],[3], which is currently being built in lake Baikal, implements in basis of preexisting developments of the neutrino telescope NT-200 [4]. The telescope records the Vavilov-Cherenkov radiation from the passage of relativistic particles in the Baikal water by a spatial lattice of photodetectors (optical modules, OMs) on scale of a cubic kilometer, focusing on neutrino interactions at energies exceeding the hundreds of TeV.

Current status of the Baikal-GVD project is reported at this conference [5]. Feature of the telescope is in his modular structure, formed from 12 functionally completed installations, called clusters, of eight vertical garlands (strings) of OMs, which are grouped on string in three sections with $12 \mathrm{OMs}$ in each, via central modules (CM or section module or ADC modules). Each cluster can operate both as a part of a whole detector and as well in an autonomous mode. The distances between garlands in cluster are about $60 \mathrm{~m}$ and between the optical modules on garlands are 15 $\mathrm{m}$. Section represents the main structural unit of data acquisition system of the telescope, providing registration of light signals, the formation of a local trigger on base of intermodular coincidence, the conversion of analog information into a digital code, and its transfer to the shore data center.

At the moment, two clusters of 288 optical modules each work in the telescope. The correct operation of such a complex system of moving detectors in a dynamically changing environment requires special procedures for the calibration and control of various parameters.

\section{LED calibration system}

An important function of the OM electronics is to provide time and amplitude calibrations of the telescope channels, which allow converting the measured signal parameters to the number of photoelectrons and signal appear time [9]. The OM calibration system is implemented based on two pulsed LED light sources and a signal generator that simulates photomultiplier signal. It is also having possibility to control the noise rate, the voltage of the photomultiplier, the power supply voltage, temperature, etc. This system is used for calibration and certification of OM before it is included in the array of the facility [8].

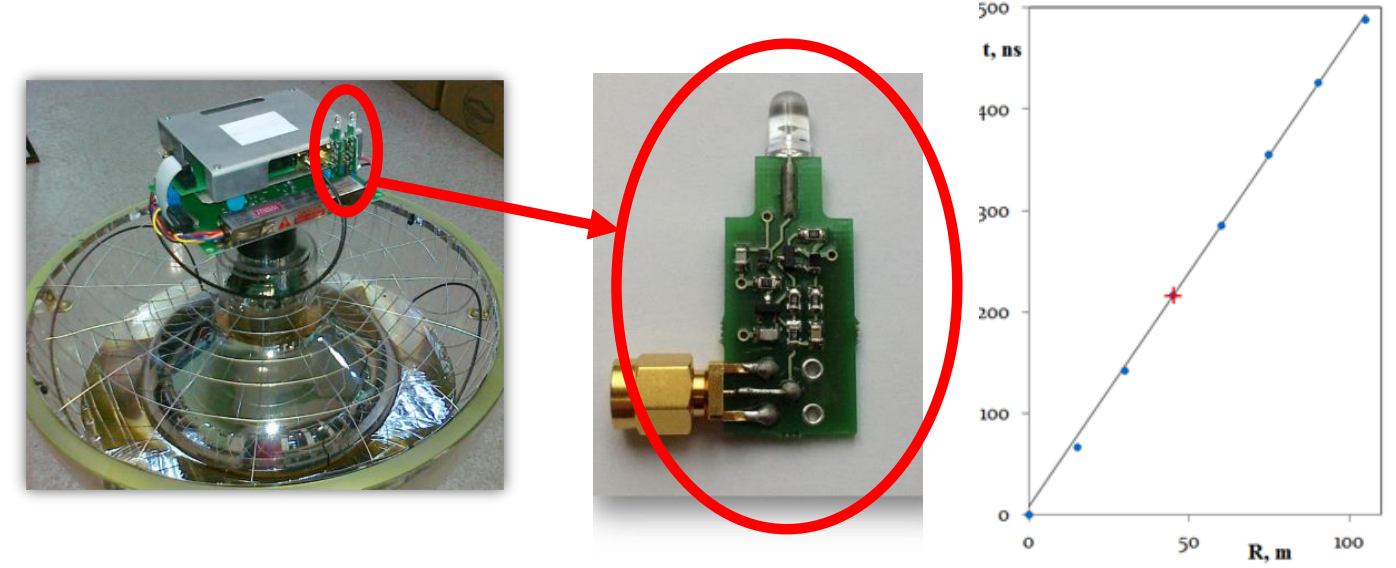

Fig.2.1 Optical module with calibration LEDs (left). LED driver with led (middle). Response time of the OM located on string at a distance above from flashing LED (right). 
When the optical module is installed, this system is used to determine the inter-channel time shifts within a one section on string. Figure 2.1 shows that the beam from such exposure can be registered by modules at a distance up to $100 \mathrm{~m}$ above the source. However, such a beam practically does not cause a trigger on neighboring garlands.

To determine the time shifts between modules of different garlands, additional sources as LED matrices are used. In the spherical deep-underwater glass case Vitrovex ${ }^{\circledR}$ with a diameter of $17 "$ are placed a controller and a set of specialized drivers with LEDs. Connection to the infrastructure of the telescope is carried out using a deep-underwater five-pin connector SubConn ${ }^{\circledR}$.
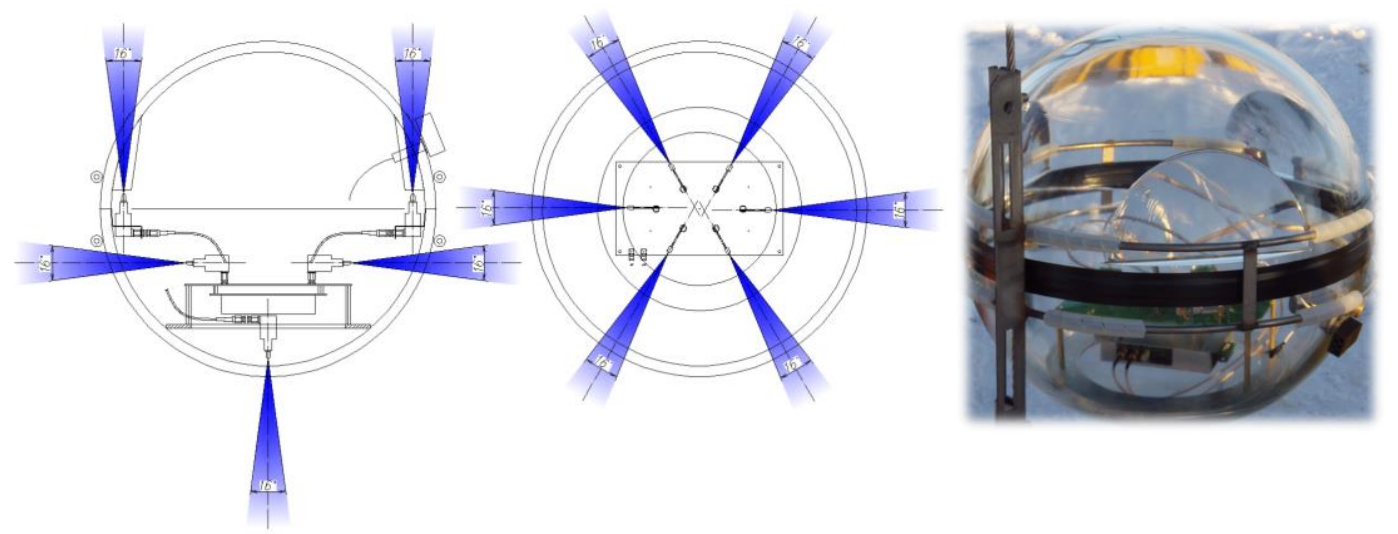

Fig.2.2 LED matrix with beam directions (left and middle) and photo of deployment (right).

In total, up to 12 LEDs ( 2 channels with 6 LEDs per channel) can be used in the matrix. In the basic configuration, six LEDs of one channel are directed horizontally with the distribution across the entire plane (60 degrees between the LEDs), and six LEDs of the other channel are directed vertically ( 2 down, through the lower pole and 4 upwards, through special prisms to minimize the influence of precipitation on the intensity of the light flux). Drivers with LEDs are connected to two special channels of the controller. Pulse overlapping of channels is less than $1 \%$. The flash intensity adjustment range for one LED is $0-10^{8}$ photons (adjustment is made independently for each channel). It is also possible to adjust the delay between the channel pulses in the range from 0 to $1000 \mathrm{~ns}$ in steps of $\sim 100 \mathrm{~ns}$ (the accuracy of setting the delay value is $\sim 1$ ns). The period between flashes is adjust from $100 \mu \mathrm{s}$ to $1 \mathrm{~s}$. The number of starts is from 1 to 65535 or in an infinite loop. The control is carried out on the RS-485 bus with addressing up to 255 nodes.

The matrix uses the Kingbright L7113 PBC-A LED model with a blue InGaN source on a $\mathrm{SiC}$ diode. The maximum flux intensity corresponds to a wavelength of $468 \mathrm{~nm}$. The duration of the light pulse depends on the batch of LEDs and amounts to $\sim 5-7 \mathrm{~ns}$. The main light flux is distributed in a cone of 16 degrees. The dependence of the number of photons on the voltage, the time distribution of the leading edge, and the distribution over the pulse width (measured with the Photonis XP1911 photomultiplier) are shown in the graphs: 

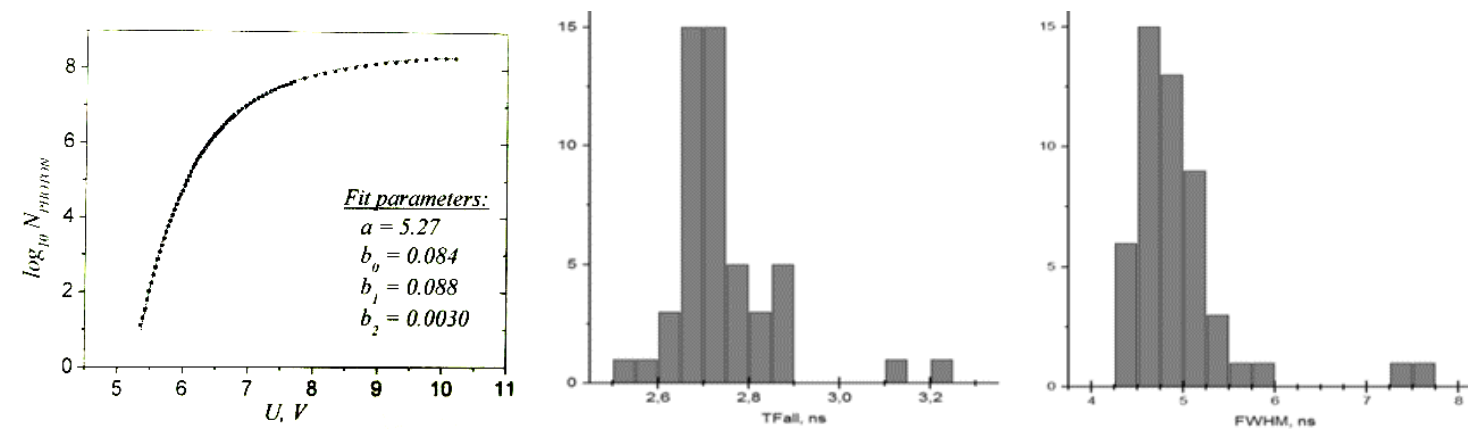

Fig.2.3 Dependence of flash photons number on LED voltage (left) and

$\mathrm{T}_{\text {fall }}$ and FWHM of pulse distribution for batch sample of LEDs.

Presently, 9 such calibration sources function in the telescope: two on the central and one on the peripheral garlands of the first cluster, three on the central cluster and two on the peripheral garlands of the second cluster, and an additional source approximately in the middle between clusters on a laser garland.

The calibration process using these sources is a special registration session with the launch of the matrices, and the storage of the data received during the session, followed by processing of the events caused by the matrix pulse.

\section{Laser sub-nanosecond pulse light source}

Another calibration device used in the Baikal-GVD is a laser light source in a deepunderwater metal case. This source can produce powerful flashes of sub-nanosecond duration, which allows for a time calibration in an inter-cluster scale. The technical drawing and photograph of the laser before dipping are presented below:
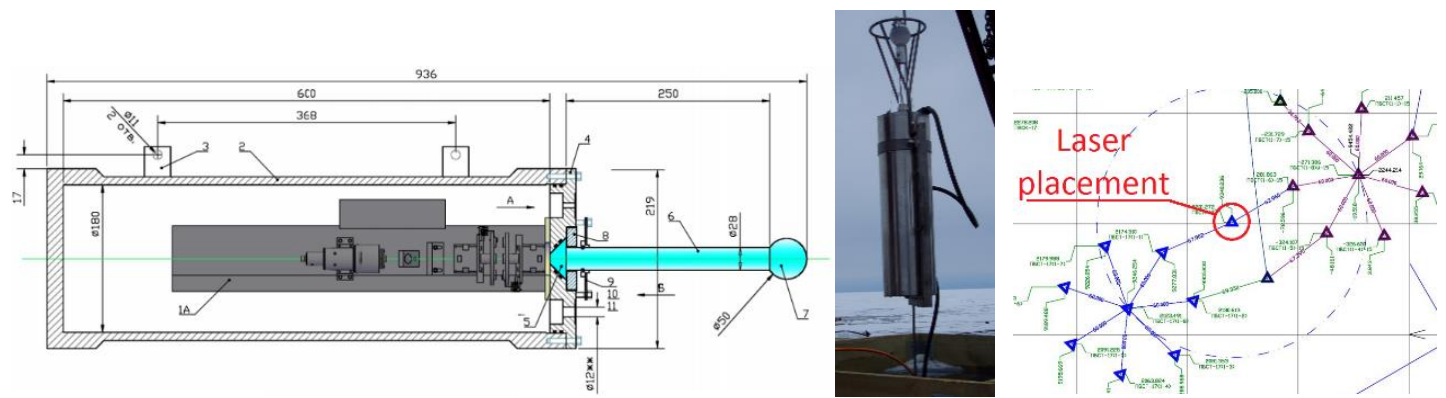

Fig.3.1 Underwater laser sub-nanosecond pulse light source, technical drawing (left), photo of deployment (middle) and placement (right).

The characteristics of the laser source are given in the following Table 3.1: 
Table 3.1 Laser light source characteristics

\begin{tabular}{lr}
\hline Main emitting element & $\begin{array}{r}\text { YAG:Nd3+ } \\
\text { (neodymium-doped yttrium aluminum garnet) }\end{array}$ \\
\hline Pulse energy & $0,37 \mathrm{~mJ}\left(\sim 10^{15}\right.$ photons per pulse) \\
\hline Instability of pulse energy per 1000 pulses & $2 \%$ \\
\hline Pulse width (FWHM) & $\sim 1 \mathrm{~ns}$ \\
\hline Number of attenuation levels & 36 \\
\hline Attenuation step & $3 \mathrm{~dB}$ \\
\hline Switching time & $<4 \mathrm{~s}$ \\
\hline Light flux change @ 1m \& angle of 300 degree & $30 \%$ \\
\hline
\end{tabular}

This unit have neodymium-doped yttrium aluminum garnet (YAG:Nd3+) As the main emitting element whose light wavelength is $1064 \mathrm{~nm}$. The beam frequency is doubled in a nonlinear crystal to a wavelength of $532 \mathrm{~nm}$. Pumping of the main element occurs due to an array of laser LEDs with a wavelength of $808 \mathrm{~nm}$. It is possible to measure the output light flux intensity by a special sensor. The attenuator is used to adjust the flash intensity. The diffuser performs the function of distributing the light flux uniformly in all directions simulating a point source. Software stuffing allows you to create and use different work scenarios, changing the number, frequency and power of light flashes during flashing session.

The block diagram of the laser source is shown at the Fig.3.2:

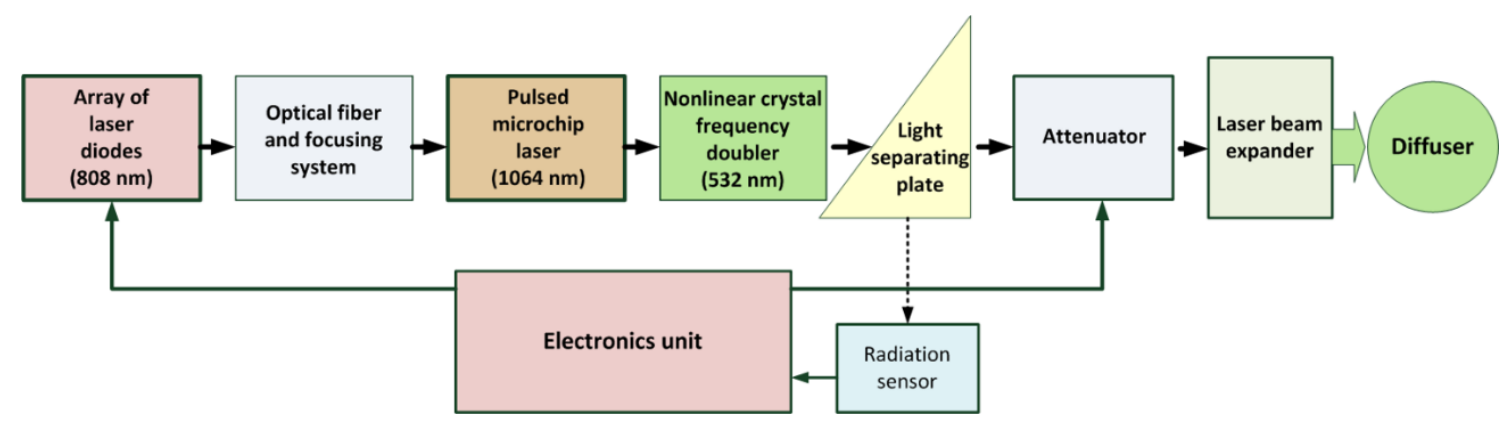

Fig.3.2 Block diagram of laser light source.

A deep-underwater laser is located approximately in the middle between two clusters at a height of 190 meters from the bottom. This position provides coverage of 85-90 percent of the optical modules of each cluster. The distribution of the amplitudes over the channels from the laser event on the first cluster is as follows: 

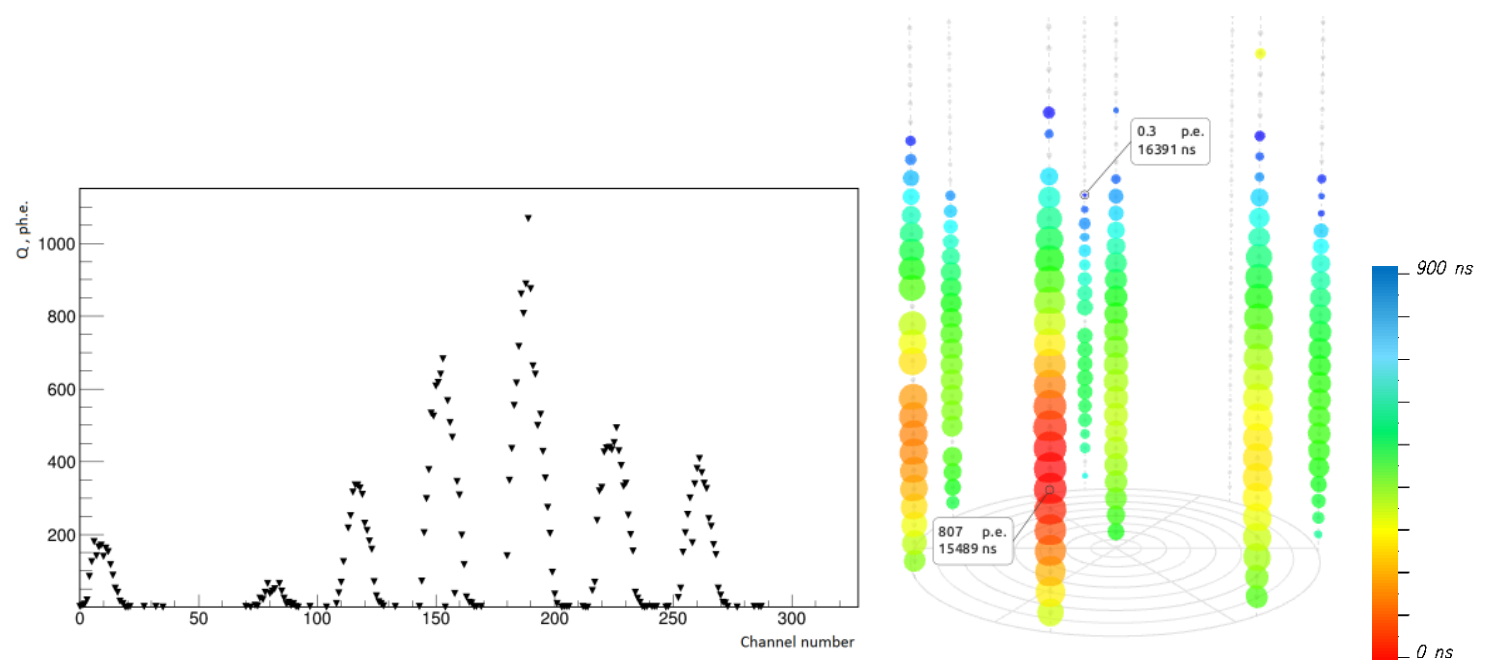

Fig.3.3 Amplitude distribution and 3d-reconstructio of "laser" event on $1^{\text {st }}$ cluster

In figure of 3D reconstruction, the diameter of the spheres is proportional to the logarithm of the number of photoelectrons on the channel. The color scale indicates the time of arrival of the pulse per channel.

\section{Monitoring system}

The monitoring system is designed to control the registration conditions of measuring channels. The principle of this system is the periodic interrogation of various parameters of the components of the telescope. It consists of the following elements: devices and sensors for measuring a set of various parameters, a system for collecting and storing data. The monitoring system equipment is partially included in the OM controller electronics and is responsible for monitoring such parameters: the PMT noise count rate, the PMT high voltage level, the OM power supply voltage, the controller temperature and it is also possible to connect a block of additional sensors via the internal $\mathrm{I}^{2} \mathrm{C}$ bus to the $\mathrm{OM}$ controller.

To control the noise count rate of the photomultiplier, a counter of nanosecond pulses is built into the OM controller. The controller allows you to adjust the duration of the time window for counting the number of noise pulses, the interval between measurements in the range from one millisecond to ten seconds, and the threshold for registering the counter. The minimum operating threshold is limited by the amplitude of the pick-up from the processor of the controller OM and amounts to $\sim 10 \mathrm{mV}(\sim 0.3$ p.e.). The following graph shows the noise levels for the fifth string of the first cluster: 


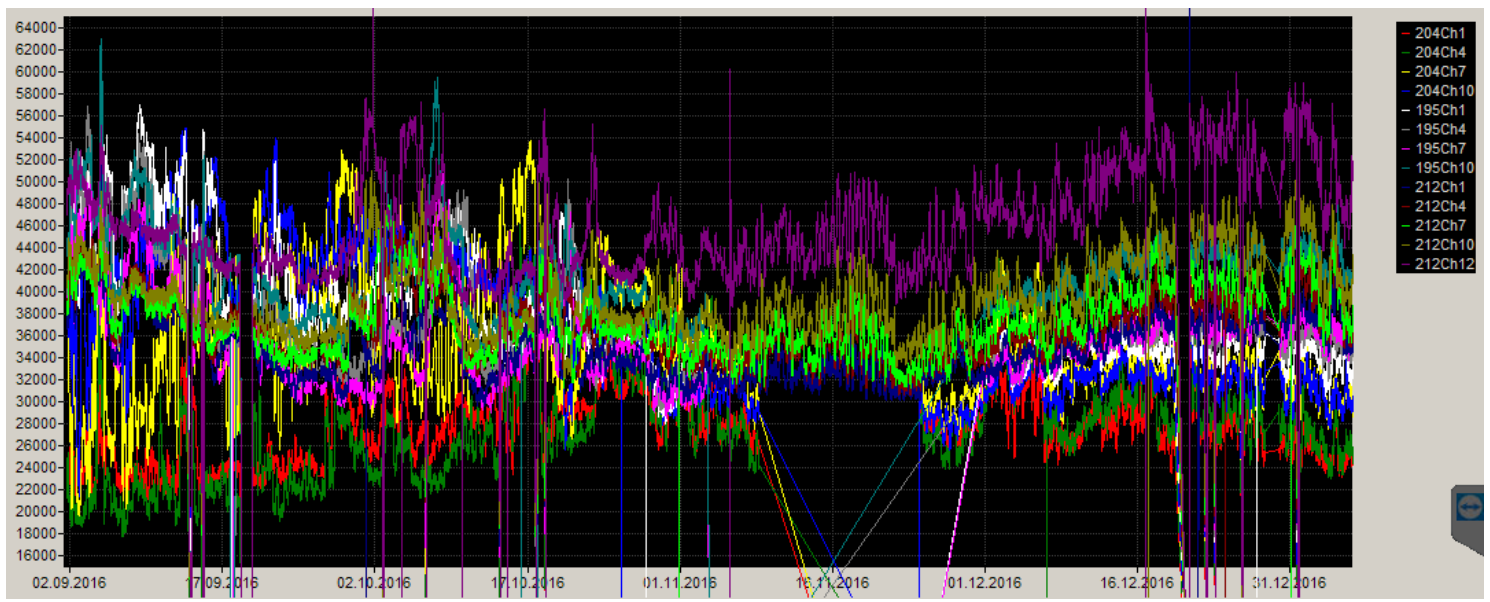

Fig.4.1 Noise in Hz of every $3^{\text {rd }}$ optical modules of $5^{\text {th }}$ string of cluster 1

In 2017, part of the modules began to contain a special unit with a set of sensors for measuring various environmental parameters: temperature, humidity, pressure and orientation parameters from a three-axis accelerometer and a magnetometer. Types and characteristics of the sensors are given in the Table 4.1:

Table 4.1 Sensors and characteristics

\begin{tabular}{|c|c|}
\hline Magnetic and acceleration 6-axis sensor LSM303 & $\begin{array}{l} \pm 2 \mathrm{~g}(\min ) \pm 16 \mathrm{~g}(\max ), \pm 1.3 \text { gauss }(\min ) \pm 8.1 \text { gauss }(\max ) \\
16 \text { bit, } 12 \text { bit (for temp.) }\end{array}$ \\
\hline Humidity sensor SHT20 & $\begin{array}{l}0 \ldots 100 \% \text { RH (typ. accur. } 3 \% \mathrm{RH}) \\
8 \text { or } 12 \text { bit (for hum.), } 12 \text { or } 14 \text { bit (for temp.) }\end{array}$ \\
\hline Pressure sensor LPS331AP & $\begin{array}{l}260 \text { to } 1260 \text { mbar absolute pressure range } \\
\text { High-resolution mode: } 0.020 \text { mbar RMS, 24-bit ADC }\end{array}$ \\
\hline Temperature Sensor MAX31725 & $\begin{array}{l}\text { Accuracy from }-40^{\circ} \mathrm{C} \text { to }+105^{\circ} \mathrm{C}: \pm 0.5^{\circ} \mathrm{C} \\
\text { Resolution: } 16 \text {-Bit }\left(0.00390625^{\circ} \mathrm{C}\right)\end{array}$ \\
\hline
\end{tabular}

At present, the monitoring system functions and records the received data in a continuous mode. Below is an example of data from the internal humidity and temperature sensor of the optical modules set:
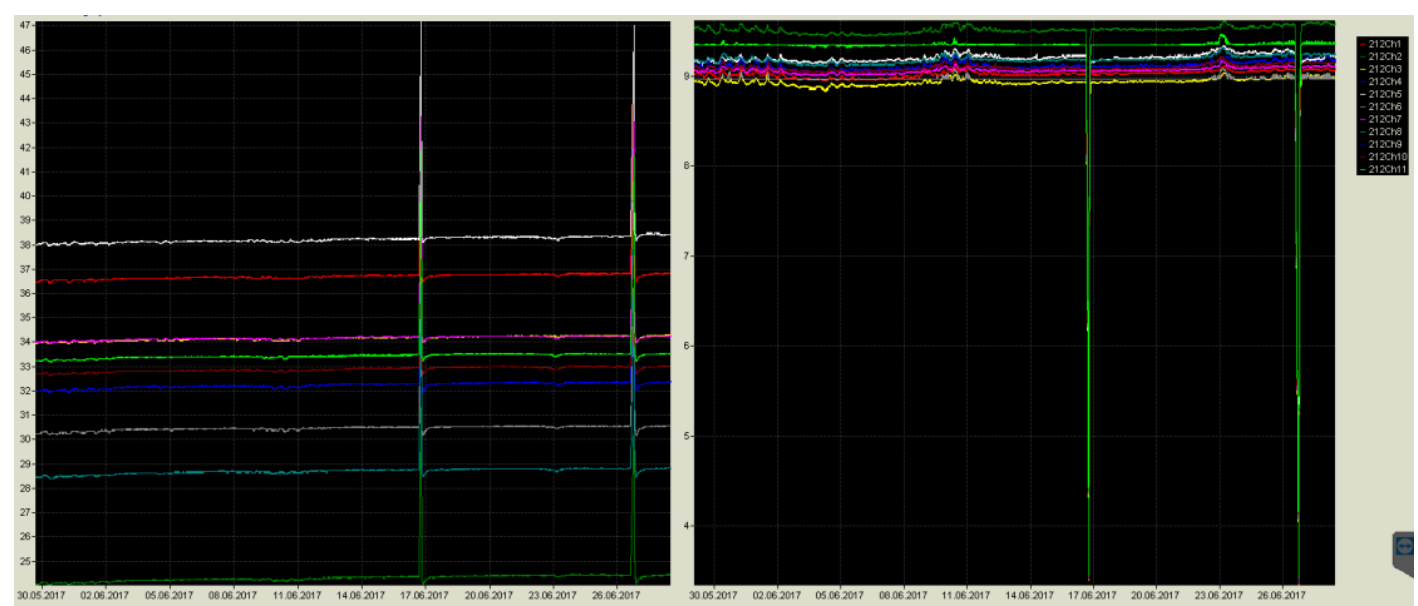

Fig.4.2 Relative humidity \% (left) and temperature ${ }^{\circ} \mathrm{C}$ (right) inside optical modules of $3^{\text {rd }}$ section of $5^{\text {th }}$ string of cluster 1 
The spread in humidity readings is explained by the difference in air humidity in the assemblage sites, the different level of saturation with the moisture of the optical gel, and the absolute error of the hygrometer readings, which can reach $10 \%$. Peaks are caused by the shutdown and, as a result, cooling the module.

\section{Conclusion}

At the moment, three different calibration systems operate in the Baikal neutrino telescope, which are one of the main ones. The availability and reliability of all three systems ensures the facility of a time calibration in full range, which is confirmed by the data obtained. Also, these systems can be easily scaled in conditions of project development [7].

The Baikal-GVD project is supported by the RFBR grants 16-29-13032, 17-02-01237.

\section{References}

[1] A. Avrorin, V. Aynutdinov, I. Belolaptikov et al., The Gigaton volume detector in lake Baikal, Nuclear Instruments and Methods in Physics Research, Section A: Accelerators, Spectrometers, Detectors and Associated Equipment 2011 vol.639 \#1 p.30-32

[2] A.V. Avrorin, A.D. Avrorin, V.M. Aynutdinov et al., The prototyping/early construction phase of the BAIKAL-GVD project, Nuclear Instruments and Methods in Physics Research, Section A: Accelerators, Spectrometers, Detectors and Associated Equipment 2014 vol.742 p.82-88.

[3] A.V. Avrorin, A.D. Avrorin, V.M. Aynutdinov et al., Baikal-GVD results, EPJ Web of Conferences 2016 vol.116 p.11005.

[4] V.M. Aynutdinov, R. Bannasch et al., The data acquisition system for Baikal-GVD, EPJ Web of Conferences 2016 vol.116 p.5004.

[5] A.V. Avrorin, A.D. Avrorin, V.M. Aynutdinov et al., Status of the Baikal-GVD experiment - 2017, $35^{\text {th }}$ International Cosmic Ray Conference 2017 (ICRC2017).

[6] V. Aynutdinov, V. Balkanov, I. Belolaptikov et al., The Baikal neutrino experiment: NT200+ and beyond, Nuclear Instruments and Methods in Physics Research, Section A: Accelerators, Spectrometers, Detectors and Associated Equipment 2007 vol.572 \#1 p.511-514.

[7] A.D. Avrorin, A.V. Avrorin, V.M. Aynutdinov et al., Status and perspectives of the BAIKAL-GVD project, EPJ Web of Conferences 2016 vol.121 p.5003.

[8] A.D. Avrorin, A.V. Avrorin, V.M. Aynutdinov et al., LED based calibration systems of the BaikalGVD neutrino telescope, EPJ Web of Conferences 2016 vol.116 p.6005.

[9] A.D. Avrorin, A.V. Avrorin, V.M. Aynutdinov et al., The optical detection unit for Baikal-GVD neutrino telescope, EPJ Web of Conferences 2016 vol.121 p.5008. 